PROCEEDINGS OF THE

AMERICAN MATHEMATICAL SOCIETY

Volume 125, Number 1, January 1997, Pages 137-144

S 0002-9939(97)03516-8

\title{
A FAMILY OF PERMITTED TRIGONOMETRIC THIN SETS
}

\author{
MIROSLAV REPICKÝ \\ (Communicated by Andreas R. Blass)
}

\begin{abstract}
We introduce the notion of perfectly measure zero sets and prove that every perfectly measure zero set is permitted for the families of all pseudoDirichlet sets, $\mathrm{N}_{0}$-sets, A-sets and $\mathrm{N}$-sets. In particular this means that these families of trigonometric thin sets are closed under adding sets of cardinality less than the additivity of Lebesgue measure.
\end{abstract}

\section{§0. INTRODUCTION}

Let $\mathcal{F}$ be a family of sets of reals. Let $A, B \in \mathcal{F}$. We say that a set $A$ is $\mathcal{F}$ permitted for $B$ if $A \cup B \in \mathcal{F}$. A set $A$ is $\mathcal{F}$-permitted if $A$ is $\mathcal{F}$-permitted for every $B \in \mathcal{F}$ (see [1]). Let $A$ be set of reals. Let us recall the following notions.

(1) $A$ is a pD-set (pseudo-Dirichlet set) if there is an increasing sequence of integers $\left\{n_{k}\right\}_{k=0}^{\infty}$ such that the sequence $\left\{\sin n_{k} \pi x\right\}_{k=0}^{\infty}$ converges quasinormally on $A$, i.e. there is a sequence of positive reals $\left\{\varepsilon_{k}\right\}_{k=0}^{\infty}$ converging to 0 such that $(\forall x \in A)\left(\forall^{\infty} k\right)\left|\sin n_{k} \pi x\right|<\varepsilon_{k}$.

(2) $A$ is an $\mathrm{N}_{0}$-set if there is an increasing sequence of integers $\left\{n_{k}\right\}_{k=0}^{\infty}$ such that $\sum_{k=0}^{\infty}\left|\sin n_{k} \pi x\right|<\infty$ for $x \in A$.

(3) $A$ is an A-set if there is an increasing sequence of integers $\left\{n_{k}\right\}_{k=0}^{\infty}$ such that $\left\{\sin n_{k} \pi x\right\}_{k=0}^{\infty}$ converges to 0 for $x \in A$.

(4) $A$ is an N-set if there is a sequence of non-negative reals $\left\{\rho_{n}\right\}_{n=0}^{\infty}$ such that $\sum_{n=0}^{\infty} \rho_{n}=\infty$ and the series $\sum_{n=0}^{\infty}\left|\rho_{n} \sin n \pi x\right|$ converges for $x \in A$.

The families of all pD-sets, $\mathrm{N}_{0}$-sets, A-sets, and $\mathrm{N}$-sets are denoted by $p \mathcal{D}, \mathcal{N}_{0}, \mathcal{A}$, and $\mathcal{N}$, respectively.

An Arbault-Erdös theorem [1] says that every countable set is $\mathcal{N}$-permitted. N. N. Kholshchevnikova [11] proved that under Martin's Axiom every set of cardinality less than $\mathfrak{c}$ is $\mathcal{N}$-permitted. L. Bukovský and Z. Bukovská [6] proved that every set of cardinality less than $\mathfrak{p}$ is $\mathcal{N}$-permitted. These results have been improved [7] by showing that every $\gamma$-set is permitted for all families of thin sets defined above. In the present paper we prove that every set having perfectly measure zero (Definition 1.1) is permitted for these families. This improves the previously mentioned results since every $\gamma$-set has perfectly measure zero. Since every set

Received by the editors February 10, 1995 and, in revised form, June 6, 1995.

1991 Mathematics Subject Classification. Primary 42A20; Secondary 03E05, 03E20.

Key words and phrases. Trigonometric thin sets, permitted sets, perfectly measure zero sets, cardinal invariants.

The work has been supported by grant 2/1224/94 of Slovenská grantová agentúra.

(C) 1997 American Mathematical Society 
of cardinality $<\operatorname{add}(\mathcal{L})$ has perfectly measure zero we obtain that every set of cardinality $<\operatorname{add}(\mathcal{L})$ is permitted.

Let us recall some further notions. The additivity of a family $\mathcal{I}$ of sets is the cardinal

$$
\operatorname{add}(\mathcal{I})=\min \left\{\left|\mathcal{I}_{0}\right|: \mathcal{I}_{0} \subseteq \mathcal{I} \& \bigcup \mathcal{I}_{0} \notin \mathcal{I}\right\}
$$

The following combinatorial characterization of the additivity of the $\sigma$-ideal $\mathcal{L}$ of Lebesgue measure zero sets is due to T. Bartoszyński [2].

Theorem 0.1. Let $S$ be an infinite countable set. Then

$$
\begin{aligned}
\operatorname{add}(\mathcal{L})=\min \left\{|\mathcal{X}|: \mathcal{X} \subseteq{ }^{\omega} S \&\right. \\
\left.\neg\left(\exists\left\{J_{n}\right\}_{n=0}^{\infty}\right)(\forall f \in \mathcal{X})\left(\forall^{\infty} n\right)\left(f(n) \in J_{n} \&\left|J_{n}\right|<n^{2}\right)\right\} .
\end{aligned}
$$

We shall need the following modification of classical Dirichlet-Minkowski Theorem (see e.g. [7]).

Theorem 0.2. Let $\left\{m_{k}\right\}_{k=0}^{\infty}$ be a strictly increasing sequence of integers. For any $j \in \omega, \delta>0$ and for any reals $x_{1}, \ldots, x_{n}$ there are integers $k_{j}, k_{j}^{\prime}$ such that $j \leq$ $k_{j}^{\prime}<k_{j} \leq j+(1 / \delta)^{n}$ and

$$
\left|\sin \left(m_{k_{j}}-m_{k_{j}^{\prime}}\right) \pi x_{l}\right|<2 \pi \delta \quad \text { for } l=1, \ldots, n .
$$

A set $A \subseteq \mathbb{R}$ has strong measure zero if for each sequence of positive reals $\left\{\varepsilon_{n}\right\}_{n=0}^{\infty}$ there is a sequence of intervals $\left\{I_{n}\right\}_{n=0}^{\infty}$ such that $\left|I_{n}\right|<\varepsilon_{n}$ for all $n$ and $A \subseteq \bigcup_{n} I_{n}$.

Let $X$ be a set of reals. A family $\mathcal{U}$ of open sets is an open $\omega$-cover of $X$ if for every finite set $X_{0} \subseteq X$ there is $U \in \mathcal{U}$ such that $X_{0} \subseteq U$. A set $X$ is a $\gamma$-set if for each open $\omega$-cover $\mathcal{U}$ of $X$ there is a sequence $\left\{U_{n}\right\}_{n=0}^{\infty}$ of elements of $\mathcal{U}$ such that $X \subseteq \bigcup_{m} \bigcap_{n \geq m} U_{n}$. The notion of a $\gamma$-set was introduced by F. Gerlits and Z. Nagy [9].

Finally let us recall that $\mathfrak{h}$ denotes the minimal cardinal such that the Boolean algebra $\mathcal{P}(\omega) /$ fin is not $\kappa$-distributive and (see [8]) $\mathfrak{p}$ is the minimal size of a family of infinite subsets of $\omega$ with finite intersection property and without infinite pseudointersection, $\mathfrak{t}$ is the minimal size of a tower on $\omega, \mathfrak{s}$ is the minimal size of a splitting family on $\omega, \mathfrak{b}$ is the minimal size of an unbounded family in ${ }^{\omega} \omega$ with the eventual ordering.

\section{$\S 1$. Perfectly measure Zero sets}

Let us introduce the main notion of the paper.

Definition 1.1. Let $A$ be a set of reals.

(i) A has perfectly measure zero if for every sequence of positive reals $\left\{\varepsilon_{n}\right\}_{n=1}^{\infty}$ there are an increasing sequence of integers $\left\{n_{k}\right\}_{k=0}^{\infty}$ and a sequence of finite families of intervals $\left\{\mathcal{I}_{n}\right\}_{n=1}^{\infty}$ such that

(1) $\left|\mathcal{I}_{n}\right| \leq n$,

(2) $|I|<\varepsilon_{n}$ for every $I \in \mathcal{I}_{n}$, and

(3) $A \subseteq \bigcup_{m} \bigcap_{k \geq m} \bigcup \mathcal{I}_{n_{k}}$.

(ii) A has uniformly measure zero if for every sequence of positive reals $\left\{\varepsilon_{n}\right\}_{n=1}^{\infty}$ there is a sequence of finite families of intervals $\left\{\mathcal{I}_{n}\right\}_{n=1}^{\infty}$ such that the above conditions (1)-(3) are satisfied for $n_{k}=k$. 
Let $\mathcal{L}_{\text {u.m.z. }}, \mathcal{L}_{\text {p.m.z. }}$, and $\mathcal{L}_{\text {s.m.z. }}$ be the family of sets having uniformly measure zero, perfectly measure zero, and strong measure zero, respectively. The next theorem summarizes several easy properties of the two families introduced in Definition 1.1 , one saying that both families are $\sigma$-ideals.

Theorem 1.2. $\quad$ (i) $\mathcal{L}_{\text {u.m.z. }} \subseteq \mathcal{L}_{\text {p.m.z. }} \subseteq \mathcal{L}_{\text {s.m.z. }}$.

(ii) Every $\gamma$-set has perfectly measure zero.

(iii) $\operatorname{add}\left(\mathcal{L}_{\text {p.m.z. }}\right) \geq \min \{\mathfrak{h}, \operatorname{add}(\mathcal{L})\}$.

(iv) $\operatorname{add}\left(\mathcal{L}_{\text {u.m.z. }}\right) \geq \operatorname{add}(\mathcal{L})$.

(vi) If a set $A \subseteq \mathbb{R}$ has uniformly measure zero or has perfectly measure zero, then the group generated by $A$ has the same property.

Proof. (i) The first inclusion is an easy consequence of definitions. We prove the second. Let a set $A$ have perfectly measure zero. Let $\left\{\varepsilon_{n}^{\prime}\right\}_{n=0}^{\infty}$ be a decreasing sequence of positive reals converging to zero. There is a sequence of finite families of intervals $\left\{\mathcal{I}_{n}\right\}_{n=1}^{\infty}$ and an increasing sequence $\left\{n_{k}\right\}_{k=0}^{\infty}$ such that conditions (1)(3) in Definition 1.1 are satisfied for the sequence of $\varepsilon_{n}=\varepsilon_{1+\cdots+n}^{\prime}$. Now let $\left\{I_{n}\right\}_{n=0}^{\infty}$ be an enumeration of $\bigcup_{n} \mathcal{I}_{n}$ so that $\left|I_{n}\right| \leq\left|I_{m}\right|$ whenever $n \geq m$. Then clearly $\left|I_{n}\right| \leq \max \left\{\varepsilon_{k}: n \leq 1+\cdots+k\right\} \leq \varepsilon_{n}^{\prime}$ and $A \subseteq \bigcup_{m} \bigcap_{k \geq m} \bigcup \mathcal{I}_{n_{k}} \subseteq \bigcap_{m} \bigcup_{n \geq m} I_{n}$.

(ii) Let $A \subseteq \mathbb{R}$ be a $\gamma$-set and let $\left\{\varepsilon_{n}\right\}_{n=1}^{\infty}$ be a given sequence of positive reals. We will find a sequence $\left\{\mathcal{I}_{n}\right\}_{n=1}^{\infty}$ and a sequence $\left\{n_{k}\right\}_{k=0}^{\infty}$ satisfying conditions (1)(3) of Definition 1.1. Without loss of generality we can assume that the sequence $\left\{\varepsilon_{n}\right\}_{n=1}^{\infty}$ is decreasing and the set $A$ is infinite. So let $\left\{y_{n}\right\}_{n=1}^{\infty}$ be a sequence of distinct elements of $A$. For every $n \geq 1$ let $\mathcal{U}_{n}$ be the family of all sets $(\bigcup \mathcal{I}) \backslash\left\{y_{n}\right\}$ where $\mathcal{I}$ is a family of at most $n$ open intervals such that $|I|<\varepsilon_{n}$ for all $I \in \mathcal{I}$. Clearly the family $\mathcal{U}=\bigcup_{n>1} \mathcal{U}_{n}$ is an open $\omega$-cover. Hence there is a sequence $\left\{U_{n}\right\}_{n=0}^{\infty}$ of elements of $\mathcal{U}$ such that $A \subseteq \bigcup_{m} \bigcap_{k \geq m} U_{k}$. Choose $n_{k}$ so that $U_{k} \in \mathcal{U}_{n_{k}}$, i.e. there is a family $\mathcal{I}_{n_{k}}$ of at most $n_{k}$ open intervals witnessing that $U_{k} \in \mathcal{U}_{n_{k}}$. In particular $U_{k} \subseteq \bigcup \mathcal{I}_{n_{k}}$. As $y_{n_{k}} \notin U_{k}$, no integer can repeat infinitely many times in the sequence $\left\{n_{k}\right\}_{k=0}^{\infty}$ and without loss of generality we can assume that this sequence is strictly increasing. For $n$ 's not appearing in the sequence $\left\{n_{k}\right\}_{k=0}^{\infty}$ take $\mathcal{I}_{n}$ arbitrary so that conditions (1)-(3) in Definition 1.1 hold true. Clearly the sequence $\left\{\mathcal{I}_{n}\right\}_{n=1}^{\infty}$ has the required properties.

(iii) Let us fix a decreasing sequence of positive reals $\left\{\varepsilon_{n}\right\}_{n=1}^{\infty}$. Let $\varepsilon_{n}^{\prime}=\varepsilon_{(n+1)^{3}}$. Let $\mathfrak{I}$ be the family of all sequences $\left\{\mathcal{I}_{n}\right\}_{n=1}^{\infty}$ of finite families of intervals with rational endpoints such that $\left|\mathcal{I}_{n}\right| \leq n$ and $|I|<\varepsilon_{n}^{\prime}$ for every $I \in \mathcal{I}_{n}$. Then for every set $A \subseteq \mathbb{R}$ having perfectly measure zero the family

$$
\mathcal{D}_{A}=\left\{a \in[\omega]^{\omega}:\left(\exists\left\{\mathcal{I}_{n}\right\}_{n=1}^{\infty} \in \mathfrak{I}\right) A \subseteq \bigcup_{m} \bigcap_{n \in a \backslash m} \bigcup \mathcal{I}_{n}\right\}
$$

is an open dense subset of $[\omega]^{\omega}, \subseteq^{*}$. To see this let $b \in[\omega]^{\omega}$ and let $\left\{b_{n}\right\}_{n=0}^{\infty}$ be an increasing enumeration of $b$. As $A$ has perfectly measure zero, there is an increasing sequence of integers $\left\{n_{k}\right\}_{k=0}^{\infty}$ and a sequence of families of intervals $\left\{\mathcal{J}_{n}\right\}_{n=1}^{\infty}$ such that $\left|\mathcal{J}_{n}\right| \leq n \leq b_{n},|I|<\varepsilon_{b_{n}}^{\prime}$ for $I \in \mathcal{J}_{n}$ and $A \subseteq \bigcup_{m} \bigcap_{k \geq m} \cup \mathcal{J}_{n_{k}}$. Let us set $a=\left\{b_{n_{k}}: k \in \omega\right\}$ and let $\left\{\mathcal{I}_{n}\right\}_{n=1}^{\infty} \in \mathfrak{I}$ be any such sequence that $\mathcal{I}_{b_{n_{k}}}=\mathcal{J}_{n_{k}}$. Clearly $\left\{\mathcal{I}_{n}\right\}_{n=1}^{\infty}$ witnesses that $a \in \mathcal{D}_{A}$.

Let $\kappa<\min \{\mathfrak{h}, \operatorname{add}(\mathcal{L})\}$ and let $\left\{A_{\xi}\right\}_{\xi<\kappa}$ be a family of sets having perfectly measure zero. As $\kappa<\mathfrak{h}$, there is a set $a \in \bigcap_{\xi<\kappa} \mathcal{D}_{A_{\xi}}$ and so for each $\xi<\kappa$ there is $\left\{\mathcal{I}_{n}^{\xi}\right\}_{n=1}^{\infty} \in \mathfrak{I}$ such that $A_{\xi} \subseteq \bigcup_{m} \bigcap_{n \in a \backslash m} \bigcup \mathcal{I}_{n}$. Since $\kappa<\operatorname{add}(\mathcal{L})$ by Theorem 0.1 , with $S=$ "the countable set of finite families of intervals with rational endpoints", 
we obtain that there exists a sequence of families of intervals $\left\{\mathcal{J}_{n}\right\}_{n=1}^{\infty}$ such that $\left|\mathcal{J}_{n}\right| \leq n^{2} \cdot n$ and for every $\xi<\kappa, \mathcal{I}_{n}^{\xi} \subseteq \mathcal{J}_{n}$ for all but finitely many $n$. Moreover we can assume that $|I|<\varepsilon_{n}^{\prime}$ for every $I \in \mathcal{J}_{n}$. Now let us set $\mathcal{I}_{n}=\mathcal{J}_{k}$ for $k^{3} \leq$ $n<(k+1)^{3}$. Then $\left|\mathcal{I}_{n}\right|=\left|\mathcal{J}_{k}\right| \leq k^{3} \leq n$ and $|I|<\varepsilon_{k}^{\prime}=\varepsilon_{(k+1)^{3}} \leq \varepsilon_{n}$ for $I \in \mathcal{I}_{n}$. Finally, for each $\xi<\kappa$ we have $A_{\xi} \subseteq \bigcup_{m} \bigcap_{n \in a \backslash m} \cup \mathcal{I}_{n}^{\xi} \subseteq \bigcup_{m} \bigcap_{n \in a \backslash m} \bigcup \mathcal{J}_{n} \subseteq$ $\bigcup_{m} \bigcap_{k \in a \backslash m} \bigcap_{n \in\left[k^{3},(k+1)^{3}\right)} \cup \mathcal{I}_{n}$. Hence $A$ has perfectly measure zero and $\operatorname{add}(\mathcal{L}) \leq$ $\min \{\mathfrak{h}, \operatorname{add}(\mathcal{L})\}$.

(iv) The proof of this part is similar to the previous one. Since $\omega \in \mathcal{D}_{A}$ whenever $A$ has uniformly measure zero, we do not need the assumption $\kappa<\mathfrak{h}$ and the above proof works with $a=\omega$. Therefore we get $\operatorname{add}(\mathcal{L}) \leq \operatorname{add}(\mathcal{L})$.

(v) Let us assume that a set $A$ has uniformly measure zero. To prove the first part of the assertion it is enough to prove that also the set $A-A=\{x-y: x, y \in A\}$ has uniformly measure zero. Let $\left\{\varepsilon_{n}\right\}_{n=1}^{\infty}$ be a sequence of positive reals. Without loss of generality we can assume that $2 \varepsilon_{n+1} \leq \varepsilon_{n}$ for all $n$. There is a sequence of families of intervals $\left\{\mathcal{J}_{n}\right\}_{n=1}^{\infty}$ such that $\left|\mathcal{J}_{n}\right| \leq n,|I|<\varepsilon_{(n+1)^{2}}$ for all $I \in \mathcal{J}_{n}$ and $A \subseteq \bigcup_{m} \bigcap_{n \geq m} \cup \mathcal{J}_{n}$, i.e.

$$
\left(\forall^{\infty} n\right) x \in \bigcup \mathcal{J}_{n} \quad \text { for } x \in A .
$$

Let $\mathcal{I}_{n}=\left\{I-J: I, J \in \mathcal{J}_{k}\right\}$ whenever $k^{2} \leq n<(k+1)^{2}$. Then $\left|\mathcal{I}_{n}\right| \leq k^{2} \leq n$ and $|I-J|<2 \varepsilon_{(k+1)^{2}} \leq \varepsilon_{n}$ for all $I, J \in \mathcal{J}_{k}$.

Let $x, y \in A$. By $(1.1)\left(\forall^{\infty} n\right) x, y \in \bigcup \mathcal{J}_{n}$ and since $\bigcup \mathcal{J}_{n}-\bigcup \mathcal{J}_{n}=\bigcup \mathcal{I}_{n^{2}}$ we have $\left(\forall^{\infty} n\right) x-y \in \bigcup \mathcal{I}_{n^{2}}$. Consequently, $A-A \subseteq \bigcup_{m} \bigcap_{n \geq m} \bigcup \mathcal{I}_{n}$.

The proof of the similar assertion for "perfectly measure zero" is the same.

In the next section we prove that every perfectly measure zero set is permitted for the families of thin trigonometric thin sets mentioned in the Introduction (definitions 1-4). Let us note that all these families are closed under group generating, while the family of strong measure zero sets need not be such since for example $\mathrm{CH}$ implies the existence of a Luzin set $X$ such that $X-X=\mathbb{R}$.

\section{§2. Perfectly measure zero sets are Permitted}

J. Arbault [1] asserted that there is a perfect $\mathcal{N}$-permitted set. Unfortunately the proof of this assertion is not correct, as N. K. Bary [5] already pointed out. We partially modify the ideas of this wrong proof and get the following results.

Theorem 2.1. Every set of reals having perfectly measure zero is $\mathcal{N}$-permitted.

Proof. Let $E \subseteq \mathbb{R}$ be an N-set. There is a sequence of non-negative reals $\left\{\rho_{n}\right\}_{n=1}^{\infty}$ such that $\sum_{n=1}^{\infty} \rho_{n}=\infty$ and $\sum_{n=1}^{\infty} \rho_{n}|\sin n \pi x|$ converges for $x \in E$. Let $s_{n}=$ $\sum_{k=1}^{n} \rho_{k}$ and let $\rho_{n}^{\prime}=\rho_{n} / s_{n}$. Clearly $\sum_{n=1}^{\infty} \rho_{n}^{\prime}=\infty$ and we can define $g(n)=$ $\min \left\{m: \sum_{k=n}^{m} \rho_{k}^{\prime} \geq 1\right\}$. Let $i: \omega \rightarrow \omega$ be a monotone unbounded function such that

$$
\sum_{n=1}^{\infty} \frac{\rho_{n}}{s_{n}^{1+1 / i(n)}}<\infty
$$

We can choose $i$ so that $\operatorname{rng}(i)=\omega \backslash\{0\}$. Let us denote $\delta_{n}=\left(1 / s_{n}\right)^{1 / i(n)}$ and let us define a sequence of positive reals $\left\{\varepsilon_{n}\right\}_{n=1}^{\infty}$ as follows:

$$
\varepsilon_{m}=\min \left\{\delta_{n}^{m+1} / n:(\exists k)[i(k)=m \& k \leq n \leq g(k)]\right\} .
$$


We prove that for every sequence of families of intervals $\left\{\mathcal{I}_{n}\right\}_{n=1}^{\infty}$ and every sequence of integers $\left\{n_{k}\right\}_{k=0}^{\infty}$ such that $\left|\mathcal{I}_{n}\right| \leq n,|I|<\varepsilon_{n}$ for $I \in \mathcal{I}_{n}$, and $g\left(n_{k}\right)<n_{k+1}$ for $k \in \omega$ we have $E \cup \bigcup_{m} \bigcap_{k \geq m} \cup \mathcal{I}_{i\left(n_{k}\right)}$ is an N-set and consequently $E \cup A \in \mathcal{N}$ for any perfectly measure zero set $A$.

Let $\mathcal{I}_{n}=\left\{\left[a_{n, l}, a_{n, l}+\varepsilon_{n}\right]\right\}_{l=1}^{n}$ be given arbitrarily, let $\left\{n_{k}\right\}_{k=0}^{\infty}$ be a sequence of integers such that $g\left(n_{k}\right)<n_{k+1}$ and let $P=\bigcup_{m} \bigcap_{k \geq m} \cup \mathcal{I}_{i\left(n_{k}\right)}$. By the DirichletMinkowski Theorem for each $k$ and for each $k \leq n \leq g(k)$ there is an integer $\lambda_{k}(n) \leq\left(1 / \delta_{n}\right)^{i(k)}$ such that

$$
\left|\sin \lambda_{k}(n) n \pi a_{i(k), l}\right| \leq 2 \pi \delta_{n} \quad \text { for } l=1, \ldots, i(k) .
$$

Since also

$$
\left|\sin \lambda_{k}(n) n \pi \varepsilon_{i(k)}\right| \leq \lambda_{k}(n) n \pi \varepsilon_{i(k)} \leq\left(1 / \delta_{n}\right)^{i(k)} n \pi \delta_{n}^{i(k)+1} / n=\pi \delta_{n},
$$

we have

$$
\left|\sin \lambda_{k}(n) n \pi x\right| \leq 3 \pi \delta_{n} \quad \text { for } x \in \bigcup \mathcal{I}_{i(k)} \text { and } k \leq n \leq g(k) .
$$

We prove that the series

$$
\sum_{k=0}^{\infty} \sum_{n=n_{k}}^{g\left(n_{k}\right)} \rho_{n}^{\prime}\left|\sin \lambda_{n_{k}}(n) n \pi x\right|
$$

converges for $x \in E \cup P$. This will finish the proof since $\sum_{k=0}^{\infty} \sum_{n=n_{k}}^{g\left(n_{k}\right)} \rho_{n}^{\prime}=\infty$.

Let $x \in P$. There is $m$ such that $x \in \bigcup \mathcal{I}_{i\left(n_{k}\right)}$ for each $k \geq m$. Then by (2.1)

$$
\sum_{k=m}^{\infty} \sum_{n=n_{k}}^{g\left(n_{k}\right)} \rho_{n}^{\prime}\left|\sin \lambda_{n_{k}}(n) n \pi x\right| \leq \sum_{k=m}^{\infty} \sum_{n=n_{k}}^{g\left(n_{k}\right)} \frac{\rho_{n}}{s_{n}} 3 \pi \delta_{n} \leq \sum_{n=n_{m}}^{\infty} \frac{3 \pi \rho_{n}}{s_{n}^{1+1 / i(n)}}<\infty .
$$

Let $x \in E$. There is $m$ such that $s_{n} \geq 1$ and so $\delta_{n} \leq 1$ for every $n \geq n_{m}$. Therefore $\lambda_{n_{k}}(n) \leq\left(1 / \delta_{n}\right)^{i\left(n_{k}\right)} \leq\left(1 / \delta_{n}\right)^{i(\bar{n})}=s_{n}$ whenever $n_{k} \leq n \leq g\left(n_{k}\right)$ and $k \geq m$. We have

$$
\begin{aligned}
\sum_{k=m}^{\infty} \sum_{n=n_{k}}^{g\left(n_{k}\right)} \rho_{n}^{\prime}\left|\sin \lambda_{n_{k}}(n) n \pi x\right| & \leq \sum_{k=m}^{\infty} \sum_{n=n_{k}}^{g\left(n_{k}\right)} \frac{\rho_{n}}{s_{n}} \lambda_{n_{k}}(n)|\sin n \pi x| \\
& \leq \sum_{n=n_{m}}^{\infty} \rho_{n}|\sin n \pi x|<\infty
\end{aligned}
$$

Hence the series (2.2) converges for $x \in P \cup E$ and consequently every perfectly measure zero set is $\mathcal{N}$-permitted.

Theorem 2.2. Every set of reals having perfectly measure zero is $p \mathcal{D}_{-}, \mathcal{N}_{0^{-}}$, and $\mathcal{A}$-permitted.

Proof. We prove only that every perfectly measure set is $\mathcal{N}_{0}$-permitted. The proofs of the remaining two cases are the same and the only difference is the convergence they deal with.

Let $E \subseteq \mathbb{R}$ be an $\mathrm{N}_{0}$-set. There is an increasing sequence of integers $\left\{m_{k}\right\}_{k=0}^{\infty}$ such that $\sum_{k=0}^{\infty}\left|\sin m_{k} \pi x\right|$ converges for $x \in E$. We can assume that the sequence $\left\{m_{k+1}-m_{k}\right\}_{k=0}^{\infty}$ is strictly increasing. Let $\delta_{n}=1 / n^{2}$ and let us define $\varepsilon_{n}=\delta_{n} / m_{k}$ for $k=\sum_{j=1}^{n}\left(1 / \delta_{j}\right)^{j}$. We prove that for every strictly increasing sequence of integers $\left\{n_{k}\right\}_{k=0}^{\infty}$ and every sequence of families of intervals $\left\{\mathcal{I}_{n}\right\}_{n=1}^{\infty}$ such that 
$\left|\mathcal{I}_{n}\right| \leq n$ and $|I|<\varepsilon_{n}$ for $I \in \mathcal{I}_{n}$ the set $E \cup \bigcup_{m} \bigcap_{k \geq m} \cup \mathcal{I}_{n_{k}}$ is an $\mathrm{N}_{0}$-set and consequently $E \cup A \in \mathcal{N}_{0}$ for every perfectly measure zero set $A$.

Let $\mathcal{I}_{n}=\left\{\left[a_{n, l}, a_{n, l}+\varepsilon_{n}\right]\right\}_{l=1}^{n}$ be given arbitrarily and let $\left\{n_{k}\right\}_{k=0}^{\infty}$ be any increasing sequence of integers. Let $P=\bigcup_{m} \bigcap_{k>m} \cup \mathcal{I}_{n_{k}}$. By Theorem 0.2 for each $n$ there are integers $l_{n}, l_{n}^{\prime}$ such that $\sum_{j=1}^{n-1}\left(1 / \delta_{j}\right)^{j} \leq l_{n}^{\prime}<l_{n} \leq \sum_{j=1}^{n}\left(1 / \delta_{j}\right)^{j}$ and

$$
\left|\sin \left(m_{l_{n}}-m_{l_{n}^{\prime}}\right) \pi a_{n, l}\right| \leq 2 \pi \delta_{n} \quad \text { for } l=1, \ldots, n .
$$

Since also

$$
\left|\sin \left(m_{l_{n}}-m_{l_{n}^{\prime}}\right) \pi \varepsilon_{n}\right| \leq\left(m_{l_{n}}-m_{l_{n}^{\prime}}\right) \pi \varepsilon_{n} \leq m_{l_{n}} \pi \varepsilon_{n} \leq \pi \delta_{n},
$$

we immediately get that

$$
\left|\sin \left(m_{l_{n}}-m_{l_{n}^{\prime}}\right) \pi x\right| \leq 3 \pi \delta_{n} \quad \text { for } x \in \bigcup \mathcal{I}_{n} .
$$

Notice that both sequences $\left\{l_{n}\right\}_{n=0}^{\infty}$ and $\left\{l_{n}^{\prime}\right\}_{n=0}^{\infty}$ are strictly increasing. We show that the series

$$
\sum_{k=0}^{\infty}\left|\sin \left(m_{l_{n_{k}}}-m_{l_{n_{k}}^{\prime}}\right) \pi x\right|
$$

converges for $x \in E \cup P$.

Let $x \in P$. There is $k_{0}$ such that for each $k \geq k_{0}, x \in \bigcup \mathcal{I}_{n_{k}}$. Then by (2.3) we have $\sum_{k=k_{0}}^{\infty}\left|\sin \left(m_{l_{n_{k}}}-m_{l_{n_{k}}^{\prime}}\right) \pi x\right| \leq \sum_{k=k_{0}}^{\infty} 3 \pi \delta_{n_{k}}<\infty$.

Let $x \in E$. Then

$$
\sum_{k=0}^{\infty}\left|\sin \left(m_{l_{n_{k}}}-m_{l_{n_{k}}^{\prime}}\right) \pi x\right| \leq \sum_{k=0}^{\infty}\left|\sin m_{l_{n_{k}}} \pi x\right|+\sum_{k=0}^{\infty}\left|\sin m_{l_{n_{k}}^{\prime}} \pi x\right|<\infty .
$$

Hence the series (2.4) converges for $x \in E \cup P$ and consequently every perfectly measure zero set is $\mathcal{N}_{0}$-permitted.

Immediately from the last two theorems and Theorem 1.2 we obtain the following.

Corollary 2.3. Every set $X$ of reals of cardinality smaller than $\max \{\mathfrak{p}, \operatorname{add}(\mathcal{L})\}$ is permitted for the families $p \mathcal{D}, \mathcal{N}_{0}, \mathcal{A}$, and $\mathcal{N}$.

Theorem 2.4. Let $\mathcal{F}$ be any of the families $p \mathcal{D}, \mathcal{N}_{0}, \mathcal{A}, \mathcal{N}$. If $\mathcal{X} \subseteq \mathcal{F}$ and $|\mathcal{X}|<\mathfrak{b}$, then there is a perfect set $P$ such that $E \cup P \in \mathcal{F}$ for each set $E \in \mathcal{X}$.

Sketch of the proof. Let $\mathcal{F}=\mathcal{N}$. For every set $E \in \mathcal{X}$ find a sequence $\left\{\varepsilon_{n}(E)\right\}_{n=1}^{\infty}$ and a function $g_{E}$ in the same way as it is described in the proof of Theorem 2.1. Since $|\mathcal{X}|<\mathfrak{b}$ there is a sequence of positive reals $\left\{\varepsilon_{n}\right\}_{n=1}^{\infty}$ and a function $g$ such that for every $E \in \mathcal{X}, \varepsilon_{n} \leq \varepsilon_{n}(E)$ and $g_{E}(n) \leq g(n)$ for all but finitely many $n$. So if $\left\{\mathcal{I}_{n}\right\}_{n=1}^{\infty}$ is a sequence of families of intervals such that $\left|\mathcal{I}_{n}\right| \leq n,|I|<\varepsilon_{n}$ for $I \in \mathcal{I}_{n}$, and $g\left(n_{k}\right)<n_{k+1}$, then by the rest of the proof of Theorem 2.1 the set $P=\bigcup_{m} \bigcap_{k \geq m} \bigcup \mathcal{I}_{n_{k}}$ is $\mathcal{N}$-permitted for all sets $E \in \mathcal{X}$.

The proof of the other cases is similar and uses ideas from the proof of Theorem 2.2 . 


\section{§3. Final notes}

T. Bartoszyński and M. Scheepers [4] have improved the result of L. Bukovský and Z. Bukovská by replacing the cardinal $\mathfrak{p}$ by $\mathfrak{t}$.

Let us note that no inequality between $\mathfrak{t}$ and $\operatorname{add}(\mathcal{L})$ is provable. In particular the estimation in Theorem 2.3 for $\mathcal{N}$ is not a consequence of the previously known results. For the consistency of $\operatorname{add}(\mathcal{L})<\mathfrak{p}$ it is enough to take the natural model for $\mathrm{MA}(\sigma$-centered $)$, which is a generic extension obtained by finite support iteration of $\sigma$-centered forcing notions. Since in the extension there are no random reals the additivity of Lebesgue measure is small there. To see the consistency of the inequality $\mathfrak{t}<\operatorname{add}(\mathcal{L})$ it is good to use the known fact that $2^{<\mathfrak{t}}=2^{\omega}$ (see e.g. [8]). So to obtain the model start with a model in which $\omega_{1}<2^{\omega}<2^{\omega_{1}}$ and add $2^{\omega}$ amoeba reals by the finite support iteration. Then in the model $\operatorname{add}(\mathcal{L})>\omega_{1}$, while $\mathfrak{t}=\omega_{1}$ since the inequality $2^{\omega}<2^{\omega_{1}}$ holds also in the extension.

Some more estimations for the size of non-permitted sets can be found in [4] and [7]. In particular every set of cardinality $<\mathfrak{s}$ is $\mathcal{A}$-permitted [7]. Like the relation of the cardinal $\mathfrak{p}$ with the notion of $\gamma$-set, this result can also be set in a topological form.

Let us say that a set $A$ of reals is an s-set if for every sequence of open sets $\left\{U_{n}\right\}_{n=0}^{\infty}$ there is an increasing sequence of integers such that $\bigcap_{m} \bigcup_{k \geq m} U_{n_{k}} \cap A=$ $\bigcup_{m} \bigcap_{k>m} U_{n_{k}} \cap A$ (or equivalently, if $A \subseteq \bigcup_{m} \bigcap_{k>m} U_{n_{k}} \cup \bigcup_{m} \bigcap_{k>m} \mathbb{R} \backslash U_{n_{k}}$ ). Easily we can observe that $\mathfrak{s}$ is the minimal size of a set which is not an s-set.

Theorem 3.1. Every s-set is $\mathcal{A}$-permitted.

Proof. Let $\left\{\sin m_{k} \pi x\right\}_{k=0}^{\infty}$ converge for $x \in E$, let $\left\{m_{k}\right\}_{k=0}^{\infty}$ be strictly increasing and let $A$ be an s-set. Let $\left\{q_{n}\right\}_{n=0}^{\infty}$ be an enumeration of the set of rational numbers. Let us denote $U_{k}=\{x \in A: \cos k \pi x>0\}$ and $V_{k, n}=\left\{x \in A: \sin k \pi x<q_{n}\right\}$. Inductively by the definition of s-set we can find a sequence $\left\{a_{n}\right\}_{n=0}^{\infty}$ of infinite subsets of $\omega$ such that

(1) $a_{n+1} \subseteq^{*} a_{n} \subseteq^{*}\left\{m_{k}: k \in \omega\right\}$

(2) $A \subseteq \bigcup_{m} \bigcap_{k \in a_{0} \backslash m} U_{k} \cup \bigcup_{m} \bigcap_{k \in a_{0} \backslash m} A \backslash U_{k}$, and

(3) $A \subseteq \bigcup_{m} \bigcap_{k \in a_{n+1} \backslash m} V_{k, n} \cup \bigcup_{m} \bigcap_{k \in a_{n+1} \backslash m} A \backslash V_{k, n}$.

Let $a$ be an infinite pseudo-intersection of the family $\left\{a_{n}: n \in \omega\right\}$ and let $\left\{n_{k}\right\}_{k=0}^{\infty}$ be the strictly increasing enumeration of the set $a$. We can assume that also the sequence $\left\{n_{k+1}-n_{k}\right\}_{k=0}^{\infty}$ is strictly increasing. We immediately obtain that both sequences $\left\{\sin n_{k} \pi x\right\}_{k=0}^{\infty}$ and $\left\{\cos n_{k} \pi x\right\}_{k=0}^{\infty}$ converge for $x \in E \cup A$ and therefore the sequence $\left\{\sin \left(n_{k+1}-n_{k}\right) \pi x\right\}_{k=0}^{\infty}$ converges to 0 for $x \in E \cup A$.

Let us show that the inequality $\mathfrak{s}<\operatorname{add}(\mathcal{L})$ is consistent with ZFC. Let $M$ be any transitive model of ZFC and let $\left\langle s_{\xi}: \xi<\omega_{1}\right\rangle$ be a generic sequence of Sacks reals over $M$ obtained by the countable support iteration. Let us denote $M_{\alpha}=$ $M\left[s_{\xi}: \xi<\alpha\right], N=M_{\omega_{1}}$. We treat $s_{\xi}$ 's as subsets of $\omega$. We show that the family $\mathcal{X}=\left\{s_{\xi}: \xi<\omega_{1}\right\}$ is a splitting family. Let $a \in N$ be any infinite subset of $\omega$. Since iterations of Sacks forcing are proper there is a countable ordinal $\alpha$ such that $a \in M_{\alpha}$. Hence without loss of generality we can assume that $a \in M$. A simple forcing density argument then shows that there is $\xi<\omega_{1}$ such that $s_{\xi} \subseteq a$. Consequently the family $\mathcal{X}$ is a splitting family in $N$. Now let us assume that the above model $M$ is a model of Martin's Axiom. Since Sacks reals preserve the base of the ideal of sets having Lebesgue measure zero and this property is preserved 
by countable support iterations (see [10] or [13]), in the model $N$ the equality $\operatorname{add}(\mathcal{L})=\mathfrak{c}$ holds true and of course the equality $\mathfrak{s}=\omega_{1}$ holds true too.

The following observations are due to Irek Recław [12]. We say that a set $A$ is null additive if for every set $B$ having Lebesgue measure zero also the set $A+B$ has Lebesgue measure zero.

Theorem 3.2 (Recław). Every set A having uniformly measure zero is null additive.

Proof. Let $A \subseteq \mathbb{R}$ have uniformly measure zero and let $B=\bigcap_{m} \bigcup_{n \geq m} I_{n}$ be a $G_{\delta}$ measure zero set where $I_{n}$ for $n \in \omega$ are intervals and $\sum_{n=0}^{\infty}\left|I_{n}\right|<\infty$. Let us denote $\delta_{k}=k^{-3}$. There is an increasing sequence $\left\{n_{k}\right\}_{k=0}^{\infty}$ such that $\sum_{n=n_{k}}^{n_{k+1}}\left|I_{n}\right|<\delta_{k}$. Let $\varepsilon_{k}=\delta_{k} / n_{k+1}$. There is a sequence of finite families of intervals $\left\{\mathcal{I}_{n}\right\}_{n=1}^{\infty}$ satisfying the conditions (1)-(2) of Definition 1.1 and such that $A \subseteq \bigcup_{m} \bigcap_{k>m} \cup \mathcal{I}_{k}$. Let us denote $B_{k}=\bigcup_{n=n_{k}}^{n_{k+1}}\left(I_{n}+\bigcup \mathcal{I}_{k}\right)$. Then $\mu\left(I_{n}+\bigcup \mathcal{I}_{k}\right) \leq k\left(\left|I_{n}\right|+\varepsilon_{k}\right)$ and so $\mu\left(B_{k}\right) \leq k \sum_{n=n_{k}}^{n_{k+1}}\left|I_{n}\right|+k n_{k+1} \varepsilon_{k} \leq k \delta_{k}+1 / k^{2}=2 / k^{2}$. As $B+A \subseteq \bigcap_{m} \bigcup_{k \geq m} B_{k}$ and $\sum_{k=0}^{\infty} \mu\left(B_{k}\right)<\infty$, we get that $B+A$ has measure zero.

T. Bartoszyński and I. Recław, assuming Martin's Axiom for $\sigma$-centered forcing notions, constructed a $\gamma$-set of size $\mathfrak{c}$ which is not strongly meager. In particular this set is not null additive and this means that in proposition (ii) of Theorem 1.2 the word "perfectly" cannot be replaced by the word "uniformly" and the equality $\mathcal{L}_{\text {u.m.z. }}=\mathcal{L}_{\text {p.m.z. }}$ is not provable. Obviously every perfectly measure zero set is meager. Since every Luzin set has strong measure zero and is not meager, the equality $\mathcal{L}_{\text {p.m.z. }}=\mathcal{L}_{\text {s.m.z. }}$ is also not provable.

\section{REFERENCES}

1. Arbault J., Sur l'ensemble de convergence absolue d'une série trigonométrique, Bull. Soc. Math. France 80 (1952), 253-317. MR 14:1080

2. Bartoszyński T., Additivity of measure implies additivity of category, Trans. Amer. Math. Soc. 281 (1984), 209-213. MR 85b:03083

3. Bartoszyński T. and Recław I., Not every $\gamma$-set is strongly meager, preprint.

4. Bartoszyński T. and Scheepers M., Remarks on sets related to trigonometric series, Topology Appl. 64 (1995), 133-140. CMP 95:15

5. Bary N. K., A Treatise on Trigonometric Series, Macmillan, New York, 1964.

6. Bukovská Z. and Bukovský L., Adding small sets to an N-set, Proc. Amer. Math. Soc. 123 (1995), 3867-3873. MR 96b:04002

7. Bukovský L., Kholshchevnikova N. N. and Repický M., Thin sets of harmonic analysis and infinite combinatorics, Real Analysis Exchange 20 (1994/95), 454-509. CMP 95:17

8. van Douwen E., The integers and topology, Handbook of Set-Theoretic Topology (K. Kunen and J. E. Vaughan, eds.), North-Holland, Amsterdam, 1984, pp. 111-167. MR 87f:54008

9. Gerlits F. and Nagy Z., Some properties of $C(X)$, part I, Topology Appl. 14 (1982), 151-161. MR 84f: 54021

10. Goldstern M., Tools for your forcing construction, Set Theory of the Reals (Ramat Gan 1991), Israel Mathematical Conference Proceedings (H. Judah, ed.), vol. 6, 1993, pp. 305-360. MR 94h:03102

11. Kholshchevnikova N. N., O neschetnykh $R$ - i N-mnozhestvakh, Mat. Zametki 38 (1985), 270277; Uncountable $R$ - and $N$-sets, Math. Notes 38 (1985), 847-851. MR 87b:03115

12. Recław I., Private communication.

13. Repický M., Goldstern-Judah-Shelah preservation theorem for countable support iterations, Fund. Math. 144 (1994), 55-72. MR 95k:03082

Mathematical Institute of Slovak Academy of Sciences, Jesenná 5, 04154 Košice, SLOVAKIA

E-mail address: repicky@kosice.upjs.sk 